\title{
Subunit Arrangement of a 2-Ketoisovalerate Ferredoxin Oxidoreductase from Thermococcus profundus Revealed by a Low Resolution X-Ray Analysis
}

\author{
Yukiko Ozawa1, Yasufumi Umena' ${ }^{2}$, Takeo Imai1, Yukio Morimoto ${ }^{3 *}$ \\ ${ }^{1}$ Department of Life Science and Graduate School of Life Science, Rikkyo (St. Paul's) University, Tokyo, Japan \\ ${ }^{2}$ Photosynthesis Research Center, Okayama University, Okayama, Japan \\ ${ }^{3}$ Research Reactor Institute, Kyoto University, Osaka, Japan \\ Email: ${ }^{*}$ morimoto@rri.kyoto-u.ac.jp
}

Received 5 August 2015; accepted 5 September 2015; published 8 September 2015

Copyright (C) 2015 by authors and Scientific Research Publishing Inc.

This work is licensed under the Creative Commons Attribution International License (CC BY).

http://creativecommons.org/licenses/by/4.0/

\begin{abstract}
2-ketoisovalerate ferredoxin oxidoreductase (VOR) is a key enzyme in hyperthermophiles catalyzing the coenzyme A-dependent oxidative decarboxylation of aliphatic amino acid-derived 2-keto acids. The enzyme purified under anaerobic conditions from a hyperthermophilic archaeon, Thermococcus profundus, is a hetero-octamer $(\alpha \beta \gamma \delta)_{2}$ consisting of four different subunits, $\alpha=45$ $\mathrm{kDa}, \beta=31 \mathrm{kDa}, \gamma=22 \mathrm{kDa}$ and $\delta=13 \mathrm{kDa}$, respectively, and it has three [4Fe-4S] clusters per $\alpha \beta \gamma \delta$-protomer, similar to other ferredoxin oxidoreductases. In the present study, the native enzyme was purified from this strain and crystallized to give rod-like crystals that were suitable for $X$-ray diffraction experiments. The crystals belonged to space group $P 4_{1} 2_{1} 2$, with unit-cell parameters $a=b=136.20 \AA, c=221.07 \AA$. Diffraction images were processed to a resolution of $3.0 \AA$. The data collected so far indicate the approximate molecular boundaries and a partial main-chain trace of the enzyme.
\end{abstract}

\section{Keywords}

Oxidoreductase, X-Ray Analysis, Iron-Sulfur Cluster

\footnotetext{
${ }^{*}$ Corresponding author.

How to cite this paper: Ozawa, Y., Umena, Y., Imai, T. and Morimoto, Y. (2015) Subunit Arrangement of a 2-Ketoisovalerate Ferredoxin Oxidoreductase from Thermococcus profundus Revealed by a Low Resolution X-Ray Analysis. Advances in Enzyme Research, 3, 75-80. http://dx.doi.org/10.4236/aer.2015.33008
} 


\section{Introduction}

An energy-producing pathway in the hyperthermophilic archaeon, Thermococcus profundus, has been proposed to degrade amino acids [1] [2]. In this pathway, amino acids produced from some peptides by peptidases are converted to their corresponding 2-keto acids by transaminases. The 2-ketoisovalerate ferredoxin oxidoreductase (VOR) catalyzes a reaction to produce acetyl CoA in the presence of coenzyme A from these 2-keto acids through oxidative decarboxylation. Pyruvate ferredoxin oxidoreductase (PFOR) is one of the well-characterized members of the ferredoxin-dependent enzyme family [3]-[5], and it has been shown to catalyze the production of acetaldehyde in the presence of coenzyme A [6]. However, the enzymes indolepyruvate ferredoxin oxidoreductase (IOR) [7], 2-ketoglutarate ferredoxin oxidoreductase (KGOR) [8] and 2-ketoisovalerate ferredoxin oxidoreductase (VOR) [9] are relatively poorly characterized, and their physiological functions have not been established experimentally [10] [11]. Four types of enzyme form a structurally related superfamily in which the enzymes each contain thiamine pyrophosphate, magnesium ions, and one to three [4Fe-4S] cluster(s) as prosthetic groups. The spatial arrangement of the cofactors in D. africanus PFOR is clarified by X-ray crystallographic analysis [4], but the three-dimensional structures of the other three types of enzyme are not yet known. In order to investigate the structure-function relationships and deduce specific substrate recognition mechanisms of these four types of enzyme (PFOR, VOR, KGOR, IOR), we report here the purification and crystallization of VOR from Thermococcus profundus and a preliminary analysis of crystallographic data collected for this enzyme.

\section{Materials and Methods}

\subsection{Isolation and Purification of Protein}

The protein was purified from the native organism, Thermococcus profundus. Isolation of the VOR has been described in a previous report [12]. Cell-free extracts were loaded onto a Q-sepharose Fast Flow (GE Healthcare) column equilibrated with buffer. After a wash step the enzyme fraction was eluted with a $\mathrm{NaCl}$ linear gradient and then applied onto a hydroxyapatite column followed by a RedTOYOPEALE (TOSOH Bioscience) column. The fractions that contained VOR alone (Figure 1(a)) in the buffer of $50 \mathrm{mM}$ Tris-HCl, pH 8, $1 \mathrm{mM}$ thiamine pyrophosphate, $1 \mathrm{mM} \mathrm{MgSO}_{4}, 0.5 \mathrm{M} \mathrm{NaCl}, 2 \mathrm{mM}$ dithiothreitol, $2 \mathrm{mM}$ sodium dithionite (SIGMA Aldrich) were concentrated with an Amicon YM50 (Merck Millipore Corporation) membrane, in which the buffer was degassed and purged oxygen by a babbling of Ar gas. Protein concentration was estimated by the method of Bradford [13] modified by use of the rose bengal (Food Red No. 105) [12].

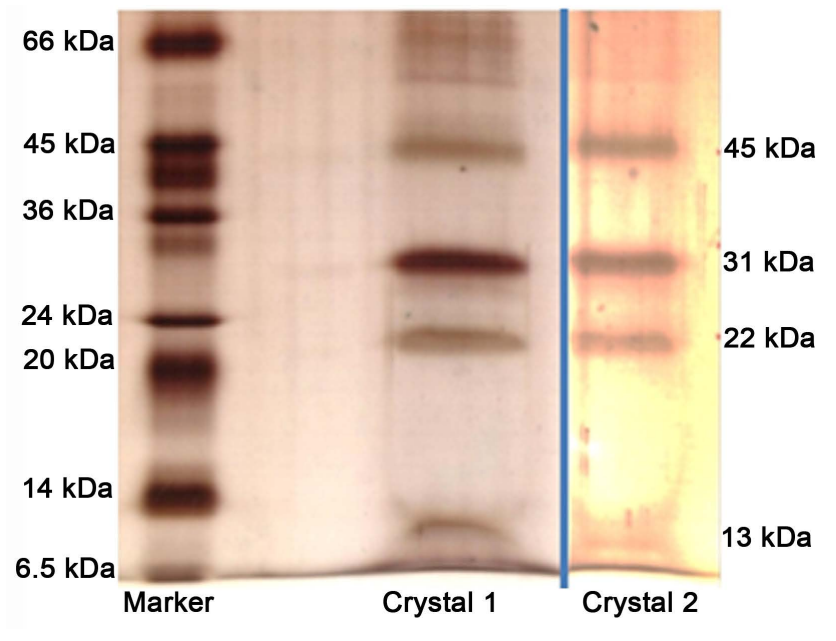

(a)

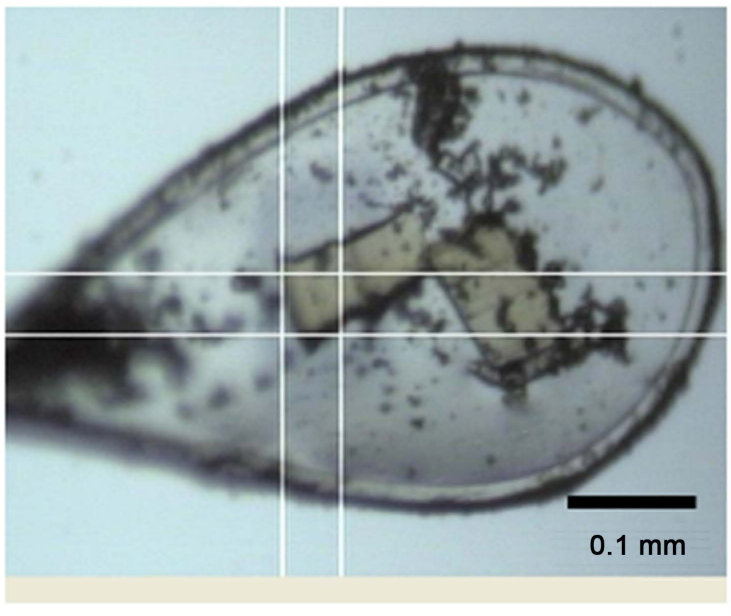

(b)

Figure 1. Purity and crystals of VOR. (a) Silver-stained SDS polyacrylamide gels showing the proteins within one dissolved crystal; left lane, molecular weight marker; middle lane, crystal 1; right lane crystal 2 (high contrast). A line is drawn between lanes showing proteins in crystal 1 and crystal 2; (b) Brownish crystals with a few stains caused by protein precipitation in a cryo-loop at the goniometer head. Scale bar is $0.1 \mathrm{~mm}$. 


\subsection{Crystallization}

Crystallization of VOR was carried out using a hanging-drop vapor diffusion method at $293 \mathrm{~K}$ under the following condition: $2 \mu \mathrm{l}$ protein solution concentrated to $10 \mathrm{mg} \cdot \mathrm{ml}^{-1}$ was mixed with $2 \mu \mathrm{l}$ reservoir solution containing 13\% (w/v) PEG6000, 0.1 M sodium cacodylate buffer at pH 6.0, $0.1 \mathrm{M} \mathrm{MgCl}_{2}, 2 \mathrm{mM}$ sodium dithionite, $10 \mathrm{mM}$ dithiothreitol and the droplet was equilibrated against $1 \mathrm{ml}$ reservoir solution (Table 1). These crystallization handling was set up in an anaerobic chamber to keep oxygen free with nitrogen gas flow. A few weeks later, crystals appeared and grew to rod-shaped crystals of $0.1 \mathrm{~mm}$ length (Figure 1(b)).

\subsection{Data Collection and Processing}

Crystals of VOR were soaked for approximately 20 - 30 sec in a cryoprotectant solution, which was the reservoir solution containing $10 \%-20 \%$ glycerol. The crystals were mounted and flash-cooled at $100 \mathrm{~K}$. Diffraction data were collected using synchrotron radiation. The diffraction data, however, were not of sufficient quality to proceed under $15 \%$ glycerol condition, because of the low resolution $(8 \AA)$ and the splitting of diffraction spots. Since crystals treated without a cryoprotectant solution diffracted well to higher resolution (4 A), data acquisition for a decision of cell parameters was carried out by enclosing the crystal in a quartz capillary with a small amount of the buffer at $288 \mathrm{~K}$. The crystal was analyzed on the BL5A at the Photon Factory (Tsukuba, Japan) and the BL44XU in the Spring-8 (Harima, Japan). After decision of cell parameters and crystal setting, a crystal treated by $20 \%$ glycerol protectant and frozen by dipping it into liquid $\mathrm{N}_{2}$ was used for high and low data acquisition. The crystal-to-detector distance was $800 \mathrm{~mm}$ for low resolution (116.0 - $5.1 \AA$ ) and $500 \mathrm{~mm}$ for high resolution (50.0 - $3.0 \AA$ ) data, and exposure times were 5 and 3 seconds, respectively. The data were collected from 1 to 180 degrees with an oscillation range of 0.5 degree (low resolution) and 1 degree (high resolution). All diffraction images were processed with the programs iMOSFLM [14] and SCALA [15] of the CCP4 program suite [16]. All data from high and low resolution data sets were merged and scaled to one data set up to $3 \AA$ resolution. The statistics of data collection and processing are summarized in Table 2 and Table 3.

\begin{tabular}{lc} 
Table 1. Crystallization condition and details. \\
\hline Method & Vapor diffusion \\
Plate type & Hanging-drop 24 wells \\
Temperature (K) & $293 \mathrm{~K}$ \\
Protein concentration & $10 \mathrm{mg} / \mathrm{ml}$ \\
Buffer composition of protein solution & $50 \mathrm{mM}$ Tris-HCl (pH 8.0)/0.2 M NaCl/4 mM DTT \\
Composition of reservoir solution & $2 \mathrm{mM}$ sodium dithionite, $10 \mathrm{mM}$ dithiothreitol \\
Volume and ratio of drop & $4 \mu \mathrm{M} 1: 1$ \\
Volume of reservoir & $1 \mathrm{ml}$ \\
\hline
\end{tabular}

Table 2. Crystallographic data and collection.

\begin{tabular}{lc}
\hline Space group & \\
Cell dimensions & \\
$a, b, c(\AA)$ & $136.20,136.20,221.07$ \\
$\alpha, \beta, \gamma\left(^{\circ}\right)$ & $90.00,90.00,90.00$ \\
Resolution $(\AA)$ & $3.00(3.16-3.00)^{\mathrm{a}}$ \\
$R_{\text {merge }}$ & $0.075(0.875)$ \\
$I / \sigma I$ & $5.0(0.5)$ \\
Completeness $(\%)$ & $100.0(99.9)$ \\
Redundancy & $3.6(2.6)$ \\
\hline
\end{tabular}

${ }^{\mathrm{a}}$ Values in parentheses are for the highest-resolution shell. 
Table 3. Refinement statistics.

\begin{tabular}{lc}
\hline Resolution $(\AA)$ & $116.0-3.00$ \\
No. reflections & 40,114 \\
$R_{\text {work }}{ }^{\mathrm{b}} / R_{\text {free }} \mathrm{c}$ & $0.3196 / 0.3674$ \\
No. atoms & 7158 \\
Protein & 0 \\
Ligand/ion & 0 \\
Water & 104.77 \\
$B$-factors & \\
Protein & \\
R.m.s. deviations & 0.170 \\
Bond lengths $(\AA)$ & 2.674 \\
Bond angles $\left({ }^{\circ}\right)$ & \\
\hline
\end{tabular}

${ }^{\mathrm{b}} \mathrm{R}_{\text {work }}$ was calculated from the working set (95\% of the data). ${ }^{\mathrm{c}} \mathrm{R}_{\text {free }}$ was calculated from the test set (5\% of the data).

\section{Results and Discussion}

The VOR crystal is a rectangular prism, crystallizing in a tetragonal crystal group with unit-cell parameters: $a=$ $b=136.20 \AA, c=221.07 \AA$. The space group, however, is uncertain because the reflection data characterizing the extinctions along the $a^{*}=b^{*}$ and $c^{*}$ axes are not recorded. A silver stained SDS electrophoresis gel of one dissolved crystal clearly showed four bands demonstrating that all four subunits were present in the crystal (Figure 1(a)). The calculated molecular weight of the VOR $\alpha \beta \gamma \delta$-protomer is $111 \mathrm{kDa}$, and the Matthews coefficient $\left(V_{\mathrm{M}}\right)$ [17] is calculated to be $2.2 \AA^{3} \mathrm{Da}^{-1}$ for sixteen molecules per unit cell. A self-rotation function calculated using low resolution data (4 $\AA$ ) from the $P 1$ space group shows definite peaks at each of the $a$-, $b$-, and $c$-axes and a diagonal position between $a$ - and $b$ - for a 2-fold symmetry with kappa $=180^{\circ}$, and also one peak along the $c$-axis for a 4-fold symmetry with kappa $=90^{\circ}$. From these results, we have concluded that the crystal corresponds to the $4 / \mathrm{mmm}$ Laue group and two $\alpha \beta \gamma \delta$-protomers exist in each asymmetric unit.

Although the amino acid sequence of the VOR from $T$. profundus has not been determined, the sequence of a homologous enzyme, VOR from $P$. furiosus, has been determined and amino acid identities are $29 \%$ ( $p f$ VOR: 299 - 664), 33\% (792 - 990), 23\% (2 - 288) (Figure 2 upper) and 27\% (699 - 747) (Figure 2 lower) against the PFOR (PDB entry 1b0p, [4]) from D. africanus. We tried to solve main-chain structures of the VOR; the PFOR was used as a model molecule for a preliminary structural analysis. A main-chain model created from the PFOR coordinates by deleting side-chain atoms of its amino acids is available for use in a molecular replacement method (MOLREP [18]), and searches of the unit cell are carried out using this model for several related space groups. A proper solution was obtained for the space group $P 4_{1} 2_{1} 2$ with values of 0.234 (Score) and 2.038 (Contrast). A preliminary structural analysis has been carried out using the program Phenix [19] and employing this space group and the PFOR main-chain model. PFOR consists of a single polypeptide chain composed of 1232 amino acids, and it appears to have four segments likely to be four domains (PFOR: 2 - 416; pfVOR: $\alpha$-subunit, 417 - 630: $\delta, 631$ - 786: $\gamma, 787-1179: \beta$ ) from its $\mathrm{N}$ - to C-terminus. The VOR structure, which is composed of four subunits $(\alpha, \beta, \gamma, \delta$, Figure 1(a)), is modified by rigid-body refinement and auto-model building using the Phenix program, with initial phases obtained from the low resolution data, producing an electron density map showing boundaries of the VOR molecule in the unit cell and assigning four subunits (Figure 3). However, the secondary structures of the chain were not yet well defined. The current $R$-factor and $R_{\text {free }}$ are 0.3196 and 0.3674 (Table 3), respectively. Searches for positions of the iron-sulfur clusters in the $\delta$ and $\beta$-subunits corresponding to the PFOR structure and of the other protomer in an asymmetric unit, and modifications of the VOR model corresponding to molecular weight of each subunit using a solvent-flattening method to improve the initial phases, are currently in progress. 


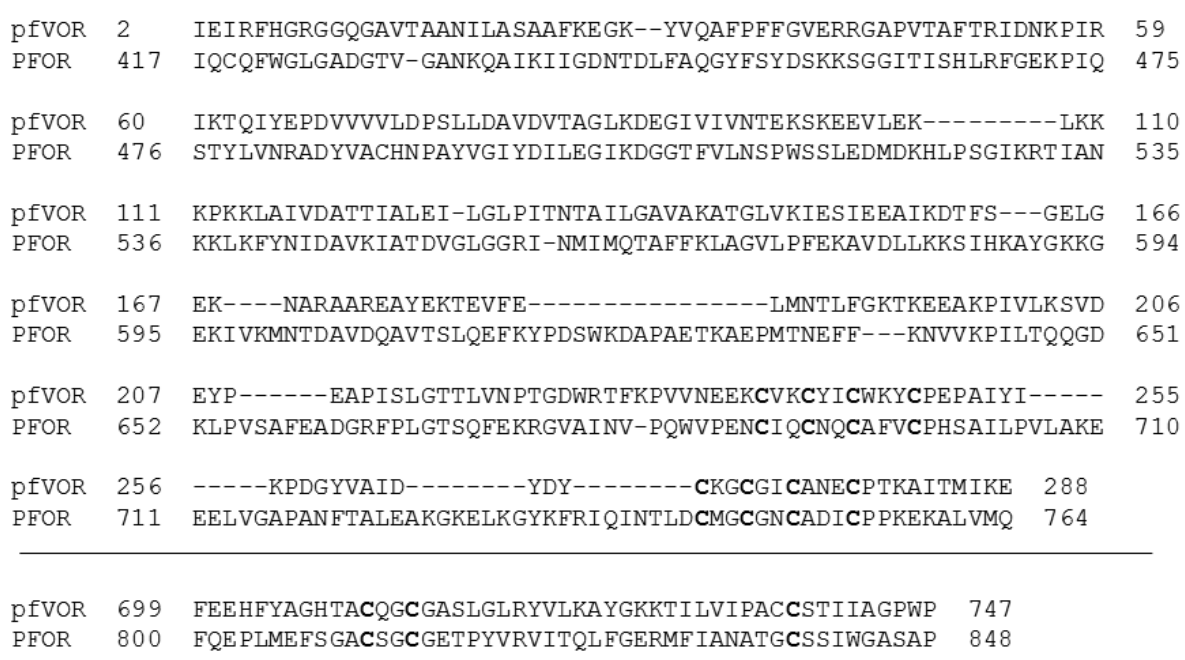

Figure 2. Amino acid alignment of the $p f$ VOR and PFOR. Cys bound Fe atoms are bold. Upper: pfVOR $\gamma \delta$-subunit, lower: a part of $\beta$-subunit.

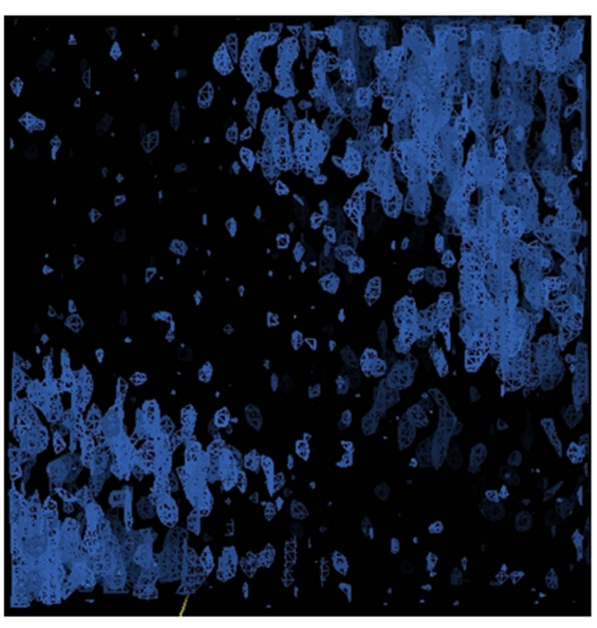

(a)

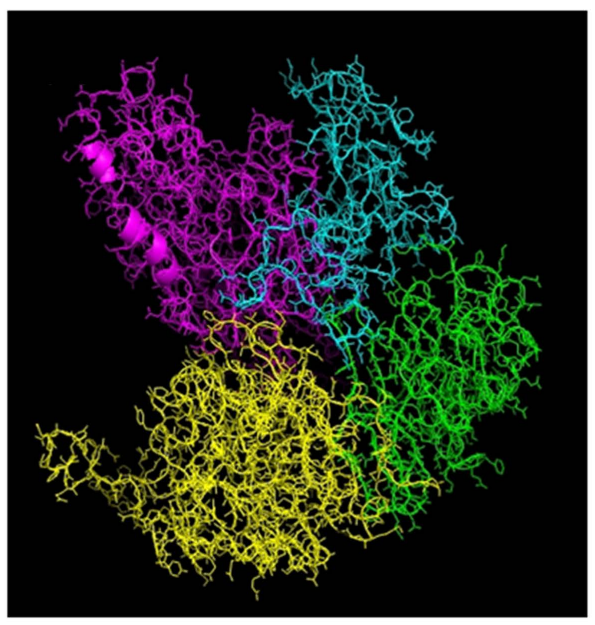

(b)

Figure 3. Electron density maps of the VOR structure at $3 \AA$ resolution; (a) boundaries of the enzyme molecules, (b) subunit arrangement of the enzyme ( $\alpha$-subunit: yellow, $\beta$ : magenta, $\gamma$ : cyan, $\delta$ : green).

\section{Acknowledgements}

This work was supported by a Grants-in-Aid for Scientific Research (22570113(YM)) from the Ministry of Education, Science and Culture of Japan and the Customer Care Plan Foundation (YM), and by a Project Research Program under approvals 25P6-1 and 23P2-5 of Research Reactor Institute, Kyoto University. The synchrotron radiation experiments were conducted under the approvals 2006AB1422 and 2011AB6647 of SPring-8 and $2006 \mathrm{G} 180$ of PF.

\section{References}

[1] Ma, K., Loessner, H.J., Heider, J., Johnson, M.K. and Adams, M.W.W. (1995) Effects of Elemental Sulfur on the Metabolism of the Deep-Sea Hyperthermophilic Archaeon Thermococcus Strain ES-1: Characterization of a Sulfur-Regulated, Non-Heme Iron Alcohol Dehydrogenase. Journal of Bacteriology, 177, 4748-4756.

[2] Heider, J., Ma, K. and Adamas, M.W.W. (1995) Purification, Characterization, and Metabolic Function of TungstenContaining Aldehyde Ferredoxin Oxidoreductase from the Hyperthermophilic and Proteolytic Archaeon Thermococcus Strain ES-1. Journal of Bacteriology, 177, 4757-4764.

[3] Adams, M.W.W. and Kletzin, A. (1996) In: Adams, M.W.W., Ed., Advances in Protein Chemistry, Vol. 48, Academic 
Press, New York, 101-180.

[4] Chabriere, E., Charon, M.-H., Volbeda, A., Pieulle, L., Hatchikian, E.C. and Fontecilla-Camps, J.C. (1999) Crystal Structures of the Key Anaerobic Enzyme Pyruvate: Ferredoxin Oxidoreductase, Free and in Complex with Pyruvate. Nature Structural Biology, 6, 182-189. http://dx.doi.org/10.1038/5870

[5] Ragsdale, S.W. (2003) Pyruvate Ferredoxin Oxidoreductase and Its Radical Intermediate. Chemical Reviews, 103, 2333-2346. http://dx.doi.org/10.1021/cr020423e

[6] Ma, K., Hutchins, A., Sung, S.J. and Adams, M.W.W. (1997) Pyruvate Ferredoxin Oxidoreductase from the Hyperthermophilic Archaeon, Pyrococcus furiosus, Functions as a CoA-Dependent Pyruvate Decarboxylase. Proceedings of the National Academy of Sciences of the United States of America, 94, 9608-9613. http://dx.doi.org/10.1073/pnas.94.18.9608

[7] Mai, X. and Adams, M.W.W. (1994) Indolepyruvate Ferredoxin Oxidoreductase from the Hyperthermophilic Archaeon Pyrococcus furiosus. A New Enzyme Involved in Peptide Fermentation. Journal of Biological Chemistry, 269, 16726-16732.

[8] Mai, X. and Adams, M.W.W. (1996) Characterization of a Fourth Type of 2-Keto Acid-Oxidizing Enzyme from a Hyperthermophilic Archaeon: 2-Ketoglutarate Ferredoxin Oxidoreductase from Thermococcus litoralis. Journal of Bacteriology, 178, 5890-5896.

[9] Heider, J., Mai, X. and Adams, M.W.W. (1996) Characterization of 2-Ketoisovalerate Ferredoxin Oxidoreductase, a New and Reversible Coenzyme A-Dependent Enzyme Involved in Peptide Fermentation by Hyperthermophilic Archaea. Journal of Bacteriology, 178, 780-787.

[10] Tersteegen, A., Linder, D., Thauer, R. and Hedderich, R. (1997) Structures and Functions of Four Anabolic 2-Oxoacid Oxidoreductases in Methanobacterium Thermoautotrophicum. European Journal of Biochemistry, 244, 862-868. http://dx.doi.org/10.1111/j.1432-1033.1997.00862.x

[11] Siddiqui, M.A., Fujiwara, S. and Imanaka, T. (1997) Indolepyruvate Ferredoxin Oxidoreductase from Pyrococcus ap. KOD1 Possesses a Mosaic Structure Showing Features of Various Oxidoreductases. Molecular and General Genetics, 254, 433-439.

[12] Ozawa, Y., Nakamura, T., Kamata, N., Yasujima, D., Urushiyama, A., Yamakura, F., Ohmori, D. and Imai, T. (2005) Thermococcus profundus 2-Ketoisovalerate Ferredoxin Oxidoreductase, a Key Enzyme in the Archaeal Energy-Producing Amino Acid Metabolic Pathway. The Journal of Biochemistry, 137, 101-107. http://dx.doi.org/10.1093/jb/mvi012

[13] Bradford, M.M. (1976) A Rapid and Sensitive Method for the Quantitation of Microgram Quantities of Protein Utilizing the Principle of Protein-Dye Binding. Analytical Biochemistry, 72, 248-254. http://dx.doi.org/10.1016/0003-2697(76)90527-3

[14] Battye, T.G.G., Kontogiannis, L., Johnson, O., Powell, H.R. and Leslie, A.G.W. (2011) iMOSFLM: A New Graphical Interface for Diffraction-Image Processing with MOSFLM. Acta Crystallographica, D67, 271-281. http://dx.doi.org/10.1107/s0907444910048675

[15] Evans, P. (2006) Scaling and Assessment of Data Quality. Acta Crystallographica, D62, 72-82. http://dx.doi.org/10.1107/s0907444905036693

[16] Potterton, E., Briggs, P., Turkenburg, M. and Dodson, E. (2003) A Graphical User Interface to the CCP4 Program Suite. Acta Crystallographica, D59, 1131-1137. http://dx.doi.org/10.1107/S0907444903008126

[17] Matthews, B.W. (1968) Solvent Content of Protein Crystals. Journal of Molecular Biology, 33, 491-497. http://dx.doi.org/10.1016/0022-2836(68)90205-2

[18] Vagin, A. and Teplyakov, A. (2010) Molecular Replacement with MOLREP. Acta Crystallographica, D66, 22-25. http://dx.doi.org/10.1107/S0907444909042589

[19] Adams, P.D., Afonine, P.V., Bunkóczi, G., Chen, V.B., Davis, I.W., Echols, N., Headd, J.J., Hung, L.-W., Kapral, G.J., Grosse-Kunstleve, R.W., McCoy, A.J., Moriarty, N.W., Oeffner, R., Read, R.J., Richardson, D.C., Richardson, J.S., Terwilliger, T.C. and Zwart, P.H. (2010) PHENIX: A Comprehensive Python-Based System for Macromolecular Structure Solution. Acta Crystallographica, D66, 213-221. http://dx.doi.org/10.1107/s0907444909052925 